\title{
Phosphorus stress induces the synthesis of novel glycolipids in Pseudomonas aeruginosa that confer protection against a last-resort antibiotic
}

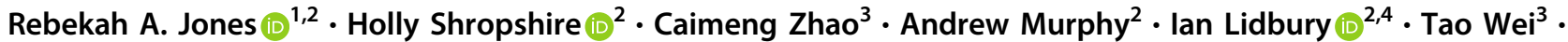 \\ David J. Scanlan $\mathbb{D}^{2} \cdot$ Yin Chen $\mathbb{D}^{2}$
}

Received: 16 February 2021 / Revised: 20 April 2021 / Accepted: 6 May 2021 / Published online: 24 May 2021

(c) The Author(s) 2021. This article is published with open access

\begin{abstract}
Pseudomonas aeruginosa is a nosocomial pathogen with a prevalence in immunocompromised individuals and is particularly abundant in the lung microbiome of cystic fibrosis patients. A clinically important adaptation for bacterial pathogens during infection is their ability to survive and proliferate under phosphorus-limited growth conditions. Here, we demonstrate that $P$. aeruginosa adapts to P-limitation by substituting membrane glycerophospholipids with sugar-containing glycolipids through a lipid renovation pathway involving a phospholipase and two glycosyltransferases. Combining bacterial genetics and multi-omics (proteomics, lipidomics and metatranscriptomic analyses), we show that the surrogate glycolipids monoglucosyldiacylglycerol and glucuronic acid-diacylglycerol are synthesised through the action of a new phospholipase (PA3219) and two glycosyltransferases (PA3218 and PA0842). Comparative genomic analyses revealed that this pathway is strictly conserved in all $P$. aeruginosa strains isolated from a range of clinical and environmental settings and actively expressed in the metatranscriptome of cystic fibrosis patients. Importantly, this phospholipid-to-glycolipid transition comes with significant ecophysiological consequence in terms of antibiotic sensitivity. Mutants defective in glycolipid synthesis survive poorly when challenged with polymyxin B, a last-resort antibiotic for treating multi-drug resistant $P$. aeruginosa. Thus, we demonstrate an intriguing link between adaptation to environmental stress (nutrient availability) and antibiotic resistance, mediated through membrane lipid renovation that is an important new facet in our understanding of the ecophysiology of this bacterium in the lung microbiome of cystic fibrosis patients.
\end{abstract}

Supplementary information The online version contains supplementary material available at https://doi.org/10.1038/s41396021-01008-7.

Yin Chen

y.chen.25@warwick.ac.uk

1 MRC Doctoral Training Partnership, University of Warwick, CV4 7AL Coventry, UK

2 School of Life Sciences, University of Warwick, CV4 7AL Coventry, UK

3 School of Food and Biological Engineering, Zhengzhou University of Light Industry, 450000 Zhengzhou, China

4 Department of Animal and Plant Sciences, University of Sheffield, Sheffield S10 2TN, UK

\section{Introduction}

Pseudomonas aeruginosa is a significant nosocomial pathogen in intensive care units causing pneumonia, surgical wound site infections and sepsis [1,2]. It is now recognised as a leading cause of morbidity and mortality in chronically infected cystic fibrosis (CF) patients and immunocompromised individuals due to the surge of carbapenem-resistant strains, a key group of first line antibiotics for treating $P$. aeruginosa infections [3]. For these drug-resistant $P$. aeruginosa strains, a viable but not ideal treatment option are polymyxins, considered to be last resort antibiotics. Although polymyxins are active against $P$. aeruginosa, their use was originally discontinued due to concerns over toxicity [4]. Indeed, $P$. aeruginosa has started to develop mechanisms of resistance to polymyxins due to an increase in their use globally. These primarily include modifications to the lipopolysaccharide (LPS) layer of the outer membrane through the addition of 4-amino-4-deoxy- 
L-arabinose $(\mathrm{L}-\mathrm{Ara} 4 \mathrm{~N})$ or phosphoethanolamine $(\mathrm{pEtN})$ $[5,6]$. These changes perturb the electrostatic interaction between cationic polymyxins and the normally negatively charged LPS.

Glycerophospholipids, such as phosphatidylglycerol (PG) and phosphatidylethanolamine (PE), are the major lipids forming the membrane lipid bilayer in bacteria, archaea, and eukaryotes [7-11]. They play a fundamental role in the evolution of the cell and it is widely accepted that the last universal common ancestor possessed a phospholipid membrane $[12,13]$. Although it is uncertain why evolution selected glycerophospholipids as the building blocks for maintaining cellular membranes [13], it is known that organisms can alter their membrane lipid composition in response to nutrient stress or environmental changes [7, 14]. Previous studies have firmly established the link between nutrient stress, particularly phosphorus availability, and the expression of a variety of virulence factors in P. aeruginosa [15-19]. However, it is unclear whether adaptation to phosphorus limitation in this bacterium causes a change in membrane lipid composition and, if so, whether lipid remodelling comes with unforeseen ecophysiological consequences. Using a synthesis of multiomics approaches, here we show that $P$. aeruginosa produces surrogate glycolipids to replace phospholipids in response to phosphorus limitation. These glycoglycerolipids have not previously been reported in $P$. aeruginosa. This lipid renovation pathway is strictly conserved in all $P$. aeruginosa strains isolated from a range of clinical settings and actively expressed in the metatranscriptome of CF patients. Importantly, such a phospholipid-to-glycolipid transition comes with a significant consequence in antibiotic sensitivity, in that glycolipids confer protection when challenged with the antimicrobial peptide polymyxin B. As such, glycolipid-mediated resistance to polymyxin $\mathrm{B}$ represents a new resistance mechanism that is quite different from the previously documented modification of LPS [5, 6]. This work highlights how the physiological adaptation of $P$. aeruginosa to phosphorus limitation can mediate a physiological response that may have profound implications for the survival of the bacteria in the lung microbiome.

\section{Materials and methods}

\section{Cultivation of $P$. aeruginosa and mutants}

P. aeruginosa strain PAO1 was obtained from the DSMZ culture collection (Germany) and routinely cultured in lysogeny broth (LB). A defined medium previously outlined for Pseudomonas species to control phosphate levels was also used [20]. This modified minimal media A comprised: Nasuccinate $20 \mathrm{mM}, \mathrm{NaCl} 200 \mathrm{mg} \mathrm{L}^{-1}, \mathrm{NH}_{4} \mathrm{Cl} 450 \mathrm{mg} \mathrm{L}^{-1}$, $\mathrm{CaCl}_{2} 200 \mathrm{mg} \mathrm{L}^{-1}, \mathrm{KCl} 200 \mathrm{mg} \mathrm{L}^{-1}, \mathrm{MgCl}_{2} 450 \mathrm{mg} \mathrm{L}^{-1}$, with trace metals $\mathrm{FeCl}_{2} 10 \mathrm{mg} \mathrm{L}^{-1}$ and $\mathrm{MnCl}_{2} 10 \mathrm{mg} \mathrm{L}^{-1}$, with $10 \mathrm{mM}$ 4-(2-hydroxyethyl)-1-piperazineethanesulfonic acid (HEPES) buffer used at $\mathrm{pH} 7 . \mathrm{Na}_{2} \mathrm{HPO}_{4}$ was then added to a final concentration of $50 \mu \mathrm{M}$ (low P) or $1 \mathrm{mM}$ (high P). An intermediate phosphate source of $400 \mu \mathrm{M} \mathrm{Na} 2 \mathrm{HPO}_{4}$ was used for overnight cultures in some experiments to prevent any excess storage of phosphate that could hamper results. All components were filter sterilised using $0.22 \mu \mathrm{m}$ pore-size filters, and made up using double deionised $\mathrm{H}_{2} \mathrm{O}$. Mutants were obtained from the $P$. aeruginosa strain PAO1 transposon mutant library at the University of Washington, and confirmed using PCR and subsequent sequencing.

\section{Alkaline phosphatase assay}

Alkaline phosphatase activity was monitored as a measure of Pi stress. Liquid $P$. aeruginosa culture samples were incubated with $10 \mathrm{mM}$ para-nitrophenol phosphate ( $p \mathrm{NPP}$ ) to a final concentration of $1 \mathrm{mM}$. Yellow- $p$ NP supernatant was measured in triplicate at $407 \mathrm{~nm}$ (BioRad iMark microplate reader). Readings were normalised using both a Tris-only incubation control and further by bacterial density (optical density reading at $600 \mathrm{~nm}\left(\mathrm{OD}_{600}\right)$ ).

\section{Over-expression of Agt1 and Agt2 in E. coli}

P. aeruginosa genes PA3218 (agt1) and PA0842 (agt2) were codon optimised for Escherichia coli and chemically synthesised (GenScript) into plasmid pET-28a(+). E. coli BLR(DE3) competent cells were thawed for 5 min before incubation with $10 \mathrm{ng}$ pET-28a_Agt plasmid, and placed on ice for $5 \mathrm{~min}$. Cells were then subjected to heat shock at $42{ }^{\circ} \mathrm{C}$ for $30 \mathrm{~s}$, placed back on ice for $2 \mathrm{~min}$. Recovery SOC media was added, with samples incubated at $37^{\circ} \mathrm{C}$ shaking, for $1 \mathrm{~h}$. Transformed cells were then plated onto kanamycinLB agar and grown overnight at $37^{\circ} \mathrm{C}$. To harvest cells for lipid extraction, single colonies were picked to grow in small volume $\mathrm{LB}-\mathrm{Kan}$ to $0.6 \mathrm{OD}_{600}$ before induction with $0.4 \mathrm{mM}$ IPTG overnight at $25^{\circ} \mathrm{C} .1 \mathrm{~mL}$ samples were then pelleted at $10,000 \times g$ for $5 \mathrm{~min}$. Pellets were stored at $-80^{\circ} \mathrm{C}$ until lipid extraction and subsequent analysis on HPLC-MS.

To purify the Agt1 and Agt2 proteins from recombinant E. coli, IPTG was added to a final concentration of $0.5 \mathrm{mM}$ once the cultures reached an $\mathrm{OD}_{600}$ of 0.6. After a further $12 \mathrm{~h}$ of growth at $30^{\circ} \mathrm{C}$, cells were harvested by centrifugation and resuspended in buffer A containing $50 \mathrm{mM}$ Tris- $\mathrm{HCl}, \mathrm{pH} 7.9,50 \mathrm{mM} \mathrm{NaCl}$. Cells were disrupted by sonication and $1 \%(\mathrm{w} / \mathrm{v})$ triton $\mathrm{X}-100$ was then added and the cells were then incubated for $2.5 \mathrm{~h}$ at $4{ }^{\circ} \mathrm{C}$. The cells were then centrifuged at $12,000 \times g$ for $20 \mathrm{~min}$, and the soluble fraction was loaded onto a nickel column (GE Healthcare, USA) pre-equilibrated with buffer A. The 
recombinant Agt1 and Agt2 enzymes were eluted with an elution buffer ( $20 \mathrm{mM}$ Tris-HCl, $\mathrm{pH} 7.9,500 \mathrm{mM} \mathrm{NaCl}$, $300 \mathrm{mM}$ imidazole) and dialysed overnight into buffer A to remove imidazole. For further purification, the samples were dialysed overnight into buffer B containing $50 \mathrm{mM}$ Tris-HCl, pH 7.9, $200 \mathrm{mM} \mathrm{NaCl}$, concentrated by ultrafiltration using a 30-kDa membrane (Millipore), and loaded onto a Superdex $200(16 / 60)$ gel filtration column (GE Healthcare, USA), which was pre-equilibrated with buffer B (50 mM Tris-HCl, $\mathrm{pH} 7.9,200 \mathrm{mM} \mathrm{NaCl}$ ). The fraction size was $0.5 \mathrm{ml}$, and the flowrate was $0.5 \mathrm{ml} / \mathrm{min}$. Purified protein was analysed by SDS-PAGE, and protein concentrations were determined using the Bradford assay.

\section{Membrane lipid extraction and HPLC-MS analysis}

Intact polar membrane lipids were extracted using a modified version of the typically used Folch extraction method [21, 22]. Liquid $P$. aeruginosa cultures growing in high and low phosphate modified minimal medium A were sampled after $8 \mathrm{~h}$, collecting the equivalent of $0.5 \mathrm{OD}_{600}$ into a $2 \mathrm{~mL}$ glass chromacol vial (Thermo Scientific), pelleted at $4{ }^{\circ} \mathrm{C}$, $4000 \mathrm{rpm}$ for $15 \mathrm{~min}$. For lipid extraction, a ratio of 500:300:1000 $\mu \mathrm{L}$ of methanol:water:chloroform (all LC-MS grade) was used. The lipid fraction was collected from the lower phase using a glass Pasteur pipette. This chloroform extract was then dried under a stream of nitrogen (Techne sample concentrator) and resuspended in $1 \mathrm{~mL} 95 \%(\mathrm{v} / \mathrm{v})$ acetonitrile (HPLC grade): $5 \%(\mathrm{w} / \mathrm{v})$ ammonium acetate (10 mM, pH 9.2) for analysis. Extracted lipid samples were analysed using an UltiMate 3000 HPLC (Thermo Scientific) system coupled to an AmazonSL quadrupole ion trap (Bruker) mass spectrometer (MS), using electrospray ionisation. Hydrophilic interaction chromatography using a BEH amide XP column (Waters) was utilised to separate lipid classes based on their head group [23]. The column chamber was maintained at $30{ }^{\circ} \mathrm{C}$ and the samples passed through at a $150 \mu \mathrm{L} \mathrm{min}{ }^{-1}$ flow rate. The mobile phase of acetonitrile:ammonium acetate $(\mathrm{pH}$ 9.2) was used to elute the sample in a 15 min per sample gradient, from 95 to $28 \%$ ammonium acetate. The lipid d17:1/12:0 sphingosylphosphoethanolamine (Sigma-Aldrich, $50 \mathrm{nM}$ ) was added to the samples and used as an internal standard. Tandem MS (or $\mathrm{MS}^{\mathrm{n}}$ ) was used to fragment the intact lipids for identification. The data were analysed using the Bruker Compass software package (DataAnalysis and QuantAnalysis).

\section{Enzyme activity assays}

The glycosyltransferase activity of Agt1 and Agt2 was measured using uridine diphosphate (UDP)-glucose or UDP-glucuronic acid and $0.1 \mathrm{mM} \mathrm{C16:0/C18:1} \mathrm{diacylgly-}$ cerol (DAG) as the substrate. $2.0 \mu \mathrm{M}$ purified enzyme was used in $10 \mathrm{mM}$ Tricine/KOH buffer, $\mathrm{pH} 8.5$ with $2 \mathrm{mM}$ dithiothreitol. The resulting mixture $(500 \mu \mathrm{l})$ was incubated at $30{ }^{\circ} \mathrm{C}$ for $60 \mathrm{~min}$ with constant shaking at $200 \mathrm{rpm}$. The lipid products were extracted using the Folch method as described above. The lipid extracts were further analysed by LC-MS for the identification of MGDG/GADG though MS $^{\mathrm{n}}$ fragmentation and for the quantification of DAG against standards. The $K_{\mathrm{m}}$ and $V_{\max }$ values were calculated using Michaelis-Menten plots with various concentrations of UDP-sugars $(0.1$ to $1.0 \mathrm{mM})$ in three replicates.

\section{Antibiotic sensitivity assays}

P. aeruginosa cultures were grown to an $\mathrm{OD}_{600}$ of 0.6 in high or low phosphate minimal media A (see above). Cultures were then diluted 1:100 in prewarmed minimal media A containing $4 \mu \mathrm{g} \mathrm{mL} \mathrm{m}^{-1}$ polymyxin B sulfate (Sigma). Samples were incubated at $37^{\circ} \mathrm{C}, 180 \mathrm{rpm}$, and assayed for survivors at specified time points by serial dilution plating onto LB. $E$. coli cultures containing pET-28a-Agt1 or pET-28a-Agt2 were grown to an $\mathrm{OD}_{600}$ of 0.6 in $\mathrm{LB}$ broth, and the expression of Agt1 and Agt2 was induced by incubation with $0.4 \mathrm{mM}$ IPTG overnight at $25^{\circ} \mathrm{C}$. A negative control of $E$. coli containing the pET-28a vector only was also set up. Overnight cultures were diluted 1:100 in prewarmed LB broth containing $0.4 \mathrm{mM}$ IPTG and $20 \mu \mathrm{g} \mathrm{mL}^{-1}$ polymyxin B sulfate (Sigma). Samples were incubated at $37^{\circ} \mathrm{C}, 180 \mathrm{rpm}$, and assayed for survivors at specified time points by serial dilution plating onto LB agar + kanamycin $25 \mu \mathrm{g} \mathrm{mL}^{-1}$.

\section{Comparative proteomic analysis}

P. aeruginosa PAO1 WT (1 $\mathrm{mM}$ phosphate, $50 \mu \mathrm{M}$ phosphate) and PlcP mutant $(50 \mu \mathrm{M}$ phosphate) cell pellets in three biological replicates were resuspended in LDS (lithium dodecyl sulfate) sample buffer containing $1 \% \beta$ mercaptoethanol before lysing at $95^{\circ} \mathrm{C}$ and vortexing. $30 \mu \mathrm{L}$ of each sample were run on NuPAGE $10 \%$ Bis-Tris protein gel (Invitrogen) for a short time before staining with SafeStain (Thermo Fisher) and excising the whole protein band. In-gel proteins were de-stained using $50 \%(\mathrm{v} / \mathrm{v})$ ethanol, $50 \mathrm{mM}$ ammonium bicarbonate (ABC), before being reduced and alkylated for $5 \mathrm{~min}$ at $70{ }^{\circ} \mathrm{C}$ using $10 \mathrm{mM}$ TCEP (tris(2-carboxyethyl)phosphine) and $40 \mathrm{mM}$ CAA (2-chloroacetamide), respectively. After washing with $50 \%$ (v/v) ethanol $50 \mathrm{mM} \mathrm{ABC}$, peptides were lysed overnight using trypsin. Finally, peptides were extracted by sonication in a water bath (10 $\mathrm{min}$ at room temperature), concentrated using a Speed-Vac (50 $\mathrm{min})$ and resuspended in $2.5 \%$ acetonitrile $0.05 \%$ formic acid. Extracted peptides were analysed by nanoLC-ESI-MS/MS using the Ultimate 3000/Orbitrap Fusion instrumentation (Thermo Scientific). The UniProt proteome for $P$. aeruginosa strain PAO1 was 
used for peptide analysis. Further data analysis was carried out using MaxQuant and Perseus software as described previously; peptides without triplicate measures were filtered out [24].

\section{Phylogenomics and metatranscriptomics analyses}

The protein sequences of PA3219, PA3218 and PA0842 were used to search genome sequences of Pseudomonas clades in the JGI IMG genome portal (https://img.jgi.doe. gov/). Note that the PA3218 protein is incorrectly annotated in the genome of PAO1. The putative glycosyltransferase located immediately downstream of PA3219 was manually inspected by aligning to the corresponding gene (PA14_22600) in the genome of $P$. aeruginosa PA14. To identify PA3218 in misannotated $P$. aeruginosa genomes, the nucleotide sequence immediately downstream of PA3219 was aligned with agt1 in strain PA14, (locus tag PA14_22600). The phylogeny of Pseudomonas clades was determined using the nucleotide sequences of six housekeeping genes (rpoB, rpoD, dnaE, recA, atpD, gyrB) retrieved from each genome using IQ-Tree with the parameters -m TEST -bb 1000 -alrt 1000. The most suitable model was chosen by the software. Evolutionary distances were inferred using maximum-likelihood analysis. Relationships were visualised using the online platform the Interactive Tree of Life viewer (https://itol.embl.de/). The conserved Pho box sequence was predicted using the MEME server [25].

The metatranscriptomics datasets of sputum samples obtained from a CF patient 7-days (SRX5145606) and 8days (SRX5145605) before death [26], CF patient G (SRR6833349) from Denmark [27] and a patient (SRR6833340) with chronic wound infection [27] were retrieved from the short reads archive (SRA) database (https://www.ncbi.nlm.nih.gov/sra). The reads were downloaded using fastq-dump and mapped using the BBMap aligner as described previously [28]. Briefly, the SRA reads were mapped to the genome sequence of $P$. aeruginosa PAO1 using a stringent cut-off of minid $=0.97$. Relative abundance data were compared using RPKM (reads per kilobase of transcript, per million mapped reads).

\section{Results and discussion}

\section{$P$. aeruginosa produces novel glycolipids in response to Pi stress}

To determine changes in the membrane lipidome in response to P-stress, the model P. aeruginosa strain PAO1 was grown in minimal medium under high $(1 \mathrm{mM})$ or low $\mathrm{Pi}(50 \mu \mathrm{M})$ conditions (Fig. 1a). The latter condition elicited strong alkaline phosphatase activity, measured through the liberation of para-nitrophenol ( $p N P$ ) from $p N P P$ (Fig. 1b), this being a strong indication that cells were P-stressed. Analysis of membrane lipid profiles using high-performance liquid chromatography coupled to mass spectrometry (HPLC-MS) revealed the presence of several new lipids under Pi stress conditions (Fig. 1c). Thus, during Pi-replete growth $(1 \mathrm{mM}$ phosphate), the lipidome is dominated by two glycerophospholipids: PG (eluted at $6.8 \mathrm{~min}$ ) and PE (eluted at $12.2 \mathrm{~min}$ ). During Pi-stress a lipid species with mass to charge ratio $(\mathrm{m} / \mathrm{z})$ of 623 and 649 were also found, with MS fragmentation resulting in a $131 \mathrm{~m} / \mathrm{z}$ peak, a diagnostic ion for the amino-acid containing ornithine lipid. This is consistent with previous reports of ornithine lipids in the $P$. aeruginosa membrane in response to Pi stress [29, 30].

Further to ornithine lipids, three unknown lipids eluting at 7.7, 8.7 and $9.8 \mathrm{~min}$, were only present under Pi stress conditions (Fig. 1c). Using several rounds of MS fragmentation $\left(\mathrm{MS}^{\mathrm{n}}\right)$, with a quadrupole ion trap MS, fragmentation patterns characteristic of glycolipids were found for all three peaks. For each peak of interest, the most predominant lipid masses of $774.7,786.8$ and $788.6 \mathrm{~m} / \mathrm{z}$ were analysed by $\mathrm{MS}^{\mathrm{n}}$ in positive ionisation mode (Fig. 1d). In each case, an initial head group was lost leaving a significant signal of $595.6 \mathrm{~m} / \mathrm{z}$, the mass of the glycolipid building block diacylglycerol (DAG). Further fragmentation leads to the loss of either fatty acyl chain from DAG, leaving monoacylglycerols of 313.2 and $339.3 \mathrm{~m} / \mathrm{z}$. Two monoacylglycerols with different masses are produced as a result of the original lipid containing 16:0 and 18:1 fatty acids $(313.2$ and $339.3 \mathrm{~m} / \mathrm{z}$ monoacylglycerols, respectively). To further elucidate the identity of the peaks, a search for a neutral loss of a polar head group was carried out. Thus, the intact masses of 774.7 and $788.6 \mathrm{~m} / \mathrm{z}$ in positive ionisation mode leads to the loss of a head group of -179 and $-193 \mathrm{~m} / \mathrm{z}$, which corresponds to a hexose- and a glucuronate- group, respectively (Fig. 1d), suggesting the occurrence of novel monoglucosyldiacylglycerol (MGDG) and glucuronic acid diacylglycerol (GADG) glycolipids in $P$. aeruginosa. The third glycolipid peak at $8.7 \mathrm{~min}$ remains an unknown lipid with intact mass of $786.8 \mathrm{~m} / \mathrm{z}$ (hereafter designated as a putative unknown glycolipid, UGL). Together, these data confirm the production of new glycolipids in $P$. aeruginosa in response to Pi stress.

\section{Comparative proteomics uncover the lipid renovation pathway in $P$. aeruginosa}

To determine the proteomic response of $P$. aeruginosa to phosphorus limitation, and identify the genes involved in glycolipid formation, strain PAO1 was cultivated under high and low Pi conditions for $8 \mathrm{~h}$ and the cellular proteome then analysed. A total of 2844 proteins were detected, 175 
(a)

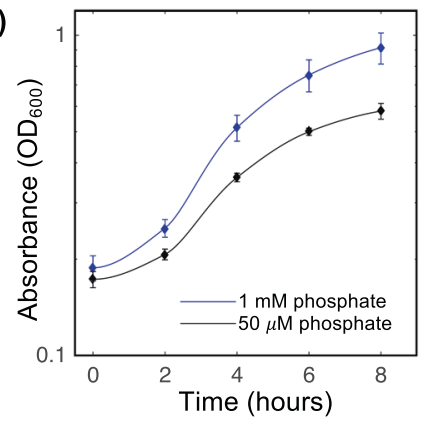

(b)

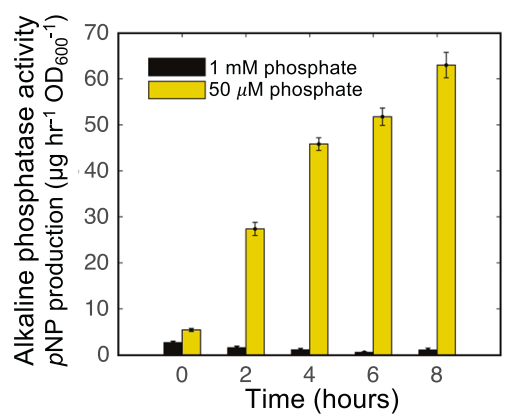

(c)

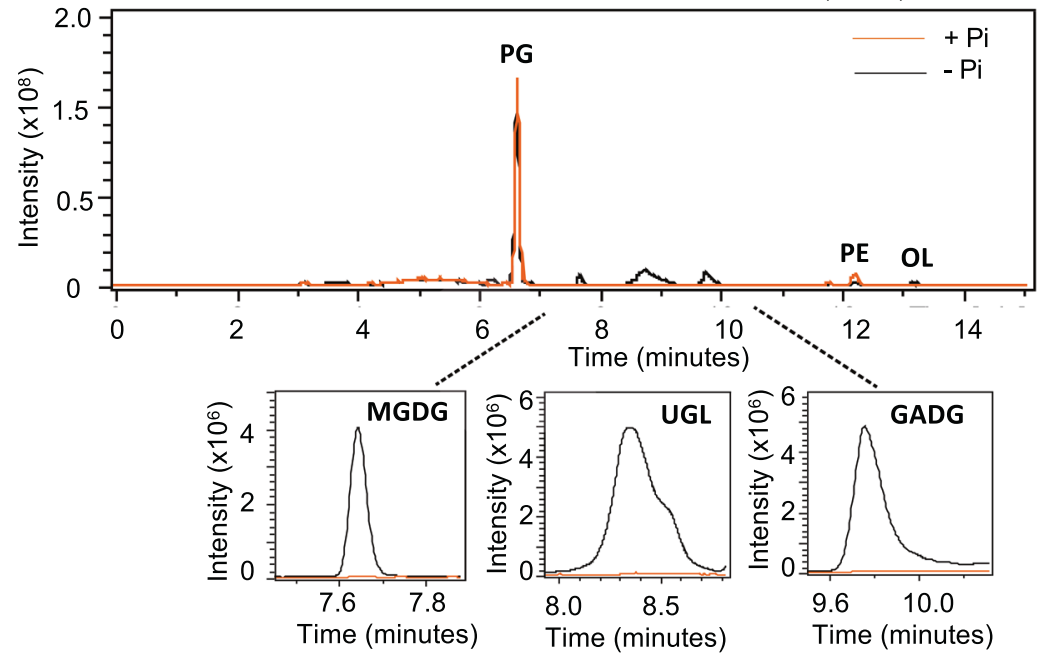

(d)
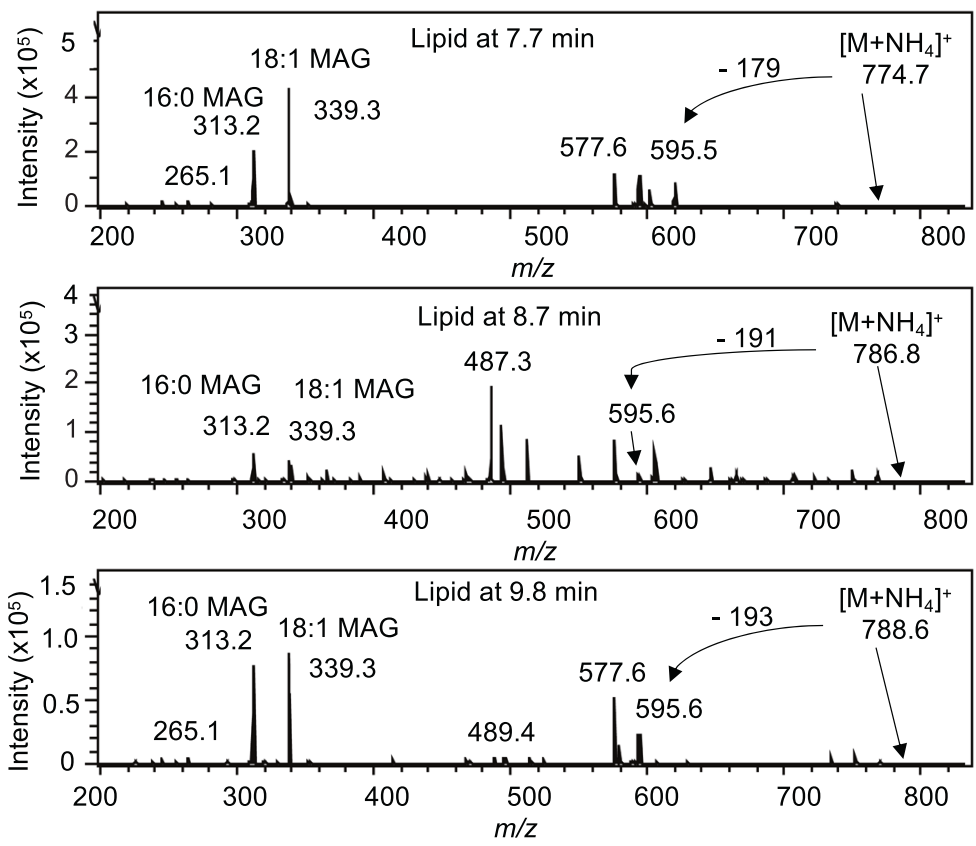

of which were found to be differentially regulated by $\mathrm{Pi}$ availability (Fig. 2a, Table S1). In line with previous transcriptomic studies of strain PAO1 [18], major phosphorus acquisition mechanisms were highly expressed under $\mathrm{Pi}$ stress conditions, e.g. the Pi-specific transporter PstSCAB, the two-component regulator PhoBR (Table S1) [31].
Comparative proteomics also identified several genes which are likely important for membrane lipid remodelling (Fig. 2b) including PA3219 (4.6-fold increase under Pideplete conditions, FDR $<0.01$ ), encoding a putative phospholipase C protein, and PA0842 (4-fold increase under Pi-deplete conditions, FDR $<0.01$ ), encoding a 
Fig. 1 Lipidomics analysis uncovers novel glycolipid formation in Pseudomonas aeruginosa strain PAO1 in response to phosphorus limitation. a Growth of strain PAO1 WT in minimal medium A containing $1 \mathrm{mM}$ phosphate $(+\mathrm{Pi}$, blue) or $50 \mu \mathrm{M}$ phosphate $(-\mathrm{Pi}$, black) over $12 \mathrm{~h}$. Data are the average of three independent replicates. b Liberation of para-nitrophenol ( $p \mathrm{NP}$ ) from para-nitrophenol phosphate $(p$ NPP) through alkaline phosphatase activity, under Pi-replete ( $1 \mathrm{mM}$, black) and Pi-deplete $(50 \mu \mathrm{M}$, yellow) conditions. Error bars represent the standard deviation of three independent replicates. c Representative chromatograms in negative ionisation mode of the $P$. aeruginosa lipidome when grown under phosphorus stress $(-\mathrm{Pi}$, black) compared to growth under phosphorus sufficient conditions (+Pi, orange). PG phosphatidylglycerol, PE phosphatidylethanolamine, OL ornithine lipids. Lower panel: extracted ion chromatograms of three new glycolipid species in $P$. aeruginosa which are only produced during Pi-limitation (black, $1 \mathrm{mM}$; orange, $50 \mu \mathrm{M}$ ). MGDG monoglucosyldiacylglycerol, GADG glucuronic acid-diacylglycerol and UGL unconfirmed glycolipid. d Mass spectrometry fragmentation spectra of three glycolipid species present under Pi stress in P. aeruginosa, at retention times of $7.7(\mathrm{~m} / \mathrm{z}, 774.7), 8.7(\mathrm{~m} / \mathrm{z}, 786.7)$ and 9.8 $(\mathrm{m} / \mathrm{z}$. 788.6) minutes, respectively. Each spectrum depicts an intact lipid mass with an ammonium $\left(\mathrm{NH}_{4}{ }^{+}\right)$adduct exhibiting neutral loss of a head group, yielding diacylglycerol (DAG) $(595 \mathrm{~m} / \mathrm{z}$ ). Further fragmentation yields monoacylglycerols (MAG) with C16:0 or C18:1 fatty acyl chains.

putative glycosyltransferase (Fig. 2a). PA3219 has 47\% protein sequence identity to PlcP from Phaeobacter sp. MED193 and 46\% identity to PlcP from Sinorhizobium meliloti [26-28]. In these bacteria, PlcP is essential in the lipid remodelling pathway for the formation of the diacylglycerol (DAG) backbone, representing the essential intermediate for the production of glycolipids [32, 33]. In $P$. aeruginosa PAO1, PA3219 appears to form an operon with PA3218, a putative glycosyltransferase likely under the control of the PhoBR two-component system, as a highly conserved Pho box sequence was recognisable in the promoter region (Fig. 2c). PA3218 (hereafter referred to as Agt1) has $41 \%$ protein sequence identity to the Agt of Phaeobacter sp. MED193. PA0842 showed 35\% identity to the Agt of Phaeobacter sp. MED193 and a Pho box sequence is also found in its promoter region. This corroborates the finding that the PA0842 protein (hereafter referred to as Agt2) was significantly upregulated under Pideplete conditions (Fig. 2a). In summary, comparative proteomic analysis suggests that $P$. aeruginosa PAO1 adopts this PlcP-Agt lipid remodelling pathway for the production of glycolipids in response to Pi-stress (Fig. 2b).

\section{The PIcP-Agt mediated lipid renovation pathway is strictly conserved in $P$. aeruginosa and actively transcribed in the metatranscriptomes of cystic fibrosis patients}

To uncover how widespread this predicted PlcP-Agt lipid remodelling pathway is amongst the genus Pseudomonas, including $P$. aeruginosa strains, we conducted a thorough comparative genomics analysis of these lipid renovating loci. PlcP-Agt is strictly conserved in all 770 genomesequenced $P$. aeruginosa strains in the IMG/M database, including all three-previously recognised $P$. aeruginosa lineages [34, 35], group 1 represented by strain PAO1, group 2 represented by strain PA14 and group 3 represented by strain PA7 (Fig. 3, Table S2). Indeed, this remodelling pathway is prevalent in many Pseudomonas groups, including the plant pathogen $P$. syringae. To investigate whether the PlcP-Agt lipid remodelling pathway is involved in host-pathogen interactions, we analysed metatranscriptomic datasets from CF patients, where $P$. aeruginosa is known to be prevalent in the fatal exacerbation period before patient death [26]. To the best of our knowledge, only two studies have reported the metatranscriptome of the bacterial community present in CF sputum [26, 27]. Indeed, phoBR and pstS are amongst the most highly expressed genes, confirming previous observations that $P$. aeruginosa is Pi-limited during human airway epithelia infection $[36,37]$. Interestingly, the alkaline phosphatase $p h o A[38]$ was highly expressed in sputum from CF patients but not from wound samples which was also dominated by $P$. aeruginosa. Importantly, the transcripts of $P$. aeruginosa agtl/plcP/agt2 are highly expressed in CF sputum during the fatal exacerbation period before death (Fig. 2d). Therefore, our phylogenomic and metatranscriptomic analyses suggest that not only is the PlcP-Agt lipid remodelling pathway strictly conserved and prevalent in P. aeruginosa, but also the corresponding genes are also highly expressed during $\mathrm{CF}$ patient infection, suggesting a potential role for lipid renovation in host-pathogen interactions.

\section{Experimental validation of the lipid renovation pathway for glycolipid formation in $P$. aeruginosa}

To validate the function of these two putative glycosyltransferases (Agt1, Agt2) in the formation of glycolipids, we synthesised the codon-optimised genes (PA3218 and PA0842, respectively) for recombinant expression in $E$. coli. The total lipidomes from the recombinant E. coli strains were then analysed by HPLC-MS to determine the presence of glycolipids in a gain-of-function assay. Expression of $P$. aeruginosa Agt1 (PA3218) was sufficient for the production of MGDG (eluted at $7.7 \mathrm{~min}$ ) in E. coli, confirmed through $\mathrm{MS}^{\mathrm{n}}$ fragmentation (Fig. 4a). No UGL nor GADG was observed in the lipidome of this Agt1overexpressing E. coli strain. Expressing Agt2 (PA0842) from $P$. aeruginosa in $E$. coli was sufficient for the accumulation of the GADG glycolipid (eluted at $9.8 \mathrm{~min}$ ), also confirmed through the $\mathrm{MS}^{\mathrm{n}}$ fragmentation pattern (Fig. 4b). Equally, no UGL nor MGDG was observed in the Agt2overexpressing E. coli strain. Production of these glycolipids was not observed in the same strain of $E$. coli 
(a)

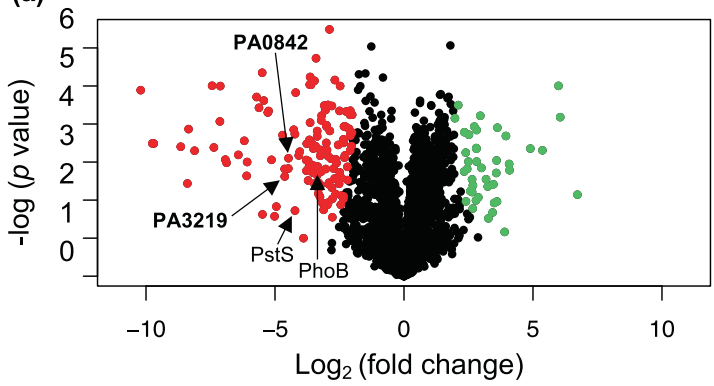

(c)

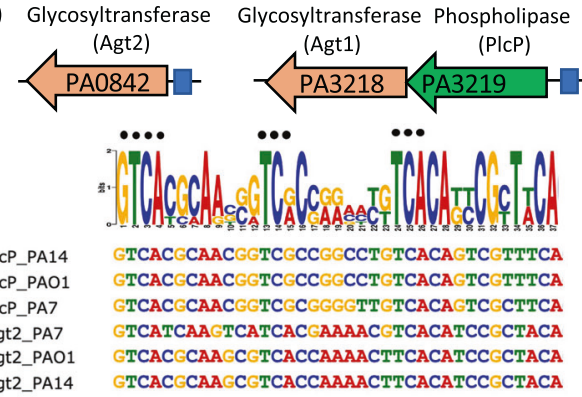

Fig. 2 Comparative multi-omic analyses for the identification of the PIcP-Agt pathway responsible for glycolipid formation in Pseudomonas aeruginosa strain PAO1. a Volcano plot depicting differentially expressed proteins when comparing Pi-replete and Pideplete conditions. Significantly upregulated proteins when under Pi stress are shown in red (left), and those that are significantly upregulated when $\mathrm{Pi}$ is sufficient are in green (right). Significance was accepted when the false discovery rate (FDR) was $<0.05$, and a fold change $\geq 2$. $\mathbf{b}$ The proposed pathway for lipid remodelling through the PlcP-Agt pathway. PlcP degrades membrane phospholipids such as PG, to generate diacylglycerol (DAG) intermediates for the formation of MGDG and GADG through the activity of glycosyltransferases, using either UDP-glucose or UDP-glucuronate as the co-substrate [41]. c Genomic organisation of predicted lipid remodelling genes in P. aeruginosa. Glycosyltransferases (orange) PA3218 (Agt1) and

transformed with an empty vector control (pET28a). It is therefore likely that UGL production is carried out by another glycosyltransferase, the identity of which remains to be discovered.

To confirm the role of Agt1 and Agt2 in the production of MGDG and GADG, we purified Agt1 and Agt2 from recombinant $E$. coli (Fig. 4c) and carried out enzyme assays using UDP-glucose and UDP-glucuronic acid as the sugar donor and DAG as the acceptor. Agt1 can only accept UDPglucose as the substrate with an affinity of $K_{\mathrm{m}}=298.1 \pm 9.5$ $\mu \mathrm{M}$ (Fig. 4c, middle panel) and produced MGDG as expected (Fig. 4d, left panel). Similarly, Agt2 can use UDPglucuronic acid as the substrate $\left(K_{\mathrm{m}}=373.0 \pm 12.9 \mu \mathrm{M}\right.$ (Fig. 4c, right panel), producing the GADG lipid (Fig. 4d, right panel). Interestingly, the purified Agt2 enzyme can also use UDP-glucose to some extent with a $K_{\mathrm{m}}$ of $480 \mu \mathrm{M}$ (data not shown) although the corresponding lipid MGDG

(d) (b)

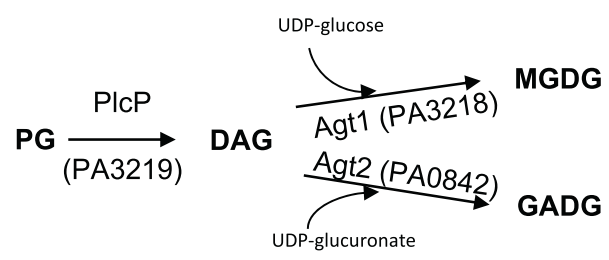

口CF D-7 aCF D-8 घCF Person G aBurn patient

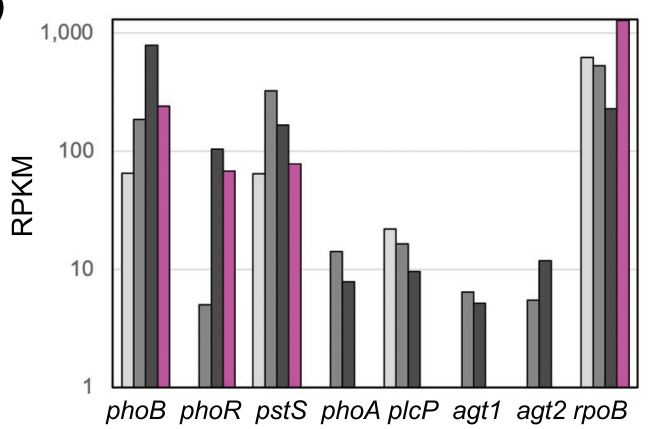

PA0842 (Agt2) are predicted to be involved in glycolipid synthesis. PA3219 is predicted to be PlcP in P. aeruginosa. Predicted Pho box sequences in the promoter regions (represented in blue boxes) of each glycosyltransferase operon from $P$. aeruginosa strains representing the PAO1 clade, the PA7 clade and the PA14 clade are shown. The black dots represent residues which are conserved in the Pho box consensus CTGTCATNNNNCTGTCAT [42]. d Metatranscriptomic analysis of PlcP-Agt lipid remodelling genes in sputum samples from a cystic fibrosis patient 7-days (CF_D-7) and 8-days (CF_D-8) before death [26] and a Danish CF patient (CF_Person G) [27] as well as a wound sample from a burns patient from the USA (Burn patient) [27]. Relative abundance is expressed as RPKM (reads per kilobase of transcript, per million mapped reads). PhoA (PA3296) encodes an alkaline phosphatase [38]. The list of RPKM abundance of individual genes of $P$. aeruginosa PAO1 is shown in Table S4.

was not observed in the lipid extract from the lipidome of the recombinant host $E$. coli (Fig. 4b).

To further confirm the role of these genes in P. aeruginosa glycolipid biosynthesis we analysed the lipidomes of mutants in $\Delta p l c P, \Delta a g t 1$ and $\Delta a g t 2$ in strain PAO1 (Fig. 5a, b). Differences were analysed by searching for the intact masses of the glycolipids MGDG and GADG: 774.6 and $788.6 \mathrm{~m} / \mathrm{z}$ in positive ionisation mode with an ammonium adduct, respectively. As expected, under Pi stress MGDG is no longer produced in the $\Delta a g t 1$ mutant and similarly GADG is no longer produced in the $\Delta a g t 2$ mutant (Fig. 5a). In the $\Delta p l c P$ mutant, no MGDG was found and the GADG lipid was significantly reduced but not entirely abolished (Fig. 5b). The small amount of GADG produced in the $\Delta p l c P$ mutant suggests that an alternative supply of DAG (independent of the degradation of phospholipids by $\mathrm{PlcP}$ ) is available in this mutant. Nevertheless, lipidome analyses 


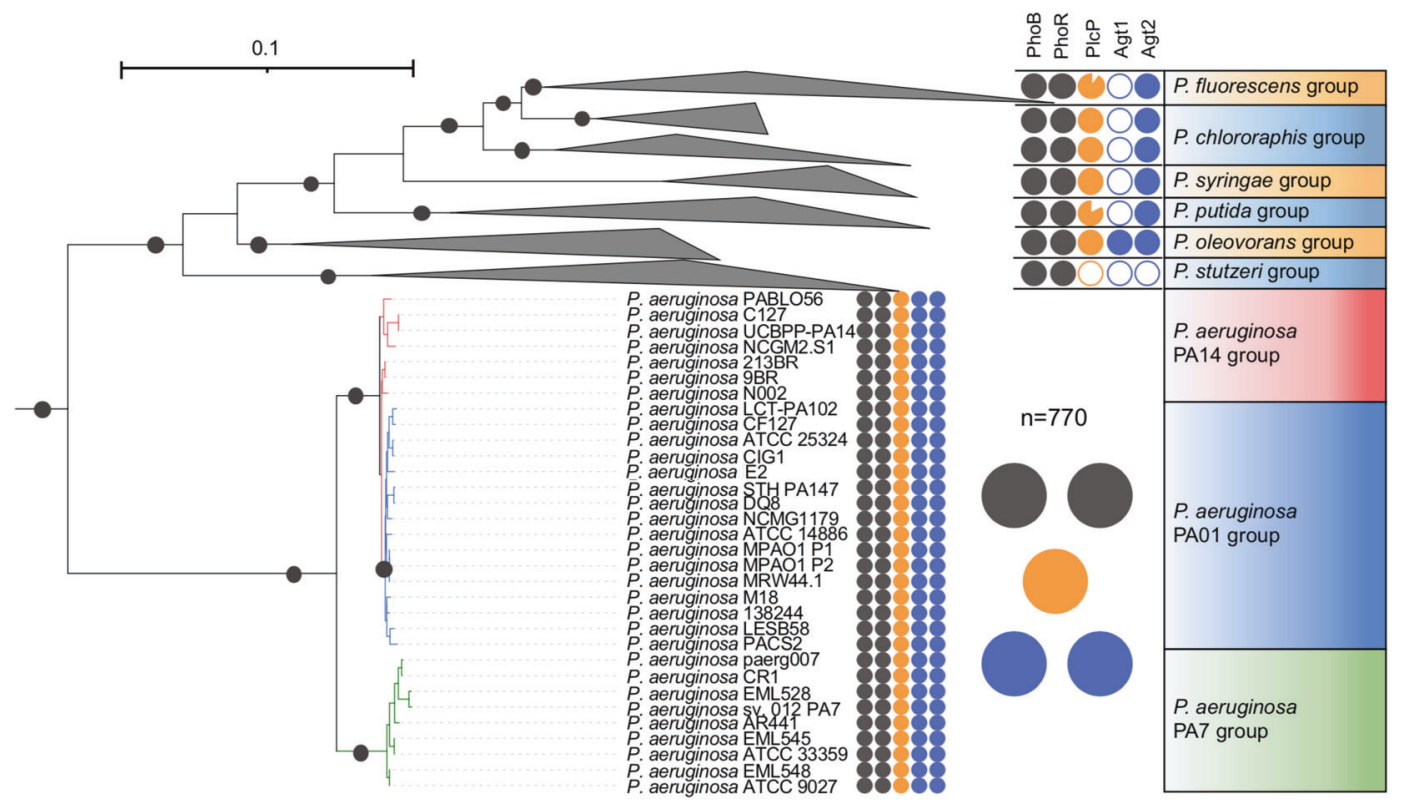

Fig. 3 Occurrence of plcP-agt genes in major Pseudomonas groups. The phylogeny of Pseudomonas clades was determined using the nucleotide sequences of six housekeeping genes $(r p o B, r p o D$, dnaE, $r e c A$, $a t p D, g y r B$ ) retrieved from each genome using IQ-Tree [43]. The filled colour indicates the presence of the genes in the genomes whereas a blank indicates the absence of the corresponding gene in the

of the $\Delta p l c P, \Delta a g t 1$ and $\Delta a g t 2$ mutants strongly supports the key role of this PlcP-Agt pathway (Fig. 2a) in lipid renovation in $P$. aeruginosa.

\section{The protective role of glycolipids to antibiotic resistance in Pseudomonas aeruginosa}

To assess whether growth of the glycolipid-deficient mutants $(\Delta p l c P, \Delta a g t 1, \Delta a g t 2)$ was affected by Pi stress, we grew the mutants in the defined minimal medium under high and low Pi conditions. However, no significant difference in growth rates was found (Fig. S1). The presence of glycolipids in the membrane may, however, have a profound impact on the functioning of the membrane during $\mathrm{Pi}$ stress. For example, PG is an anionic lipid with net negative charges whereas MGDG has a neutral charged sugar group. Although a PG-to-GADG substitution may not necessarily change membrane charge [23], it may affect membrane curvature and the packing density of lipids. Thus, subsequent knock-on effects in membrane function might be expected [10]. We therefore set out to investigate whether membrane lipid composition may have an impact on antibiotic resistance in $P$. aeruginosa. As cationic antimicrobial peptides directly interact with bacterial cell membranes, we focused on the impact of lipid remodelling on the killing activity of polymyxin $\mathrm{B}$. We conducted the analyses under P-deplete conditions, since Pi-stress is clinically important, already known to induce the genomes. The two-component system PhoBR (black circles) is found in all genomes and the PlcP-Agt1/Agt2 are strictly conserved in all 770 genome-sequenced $P$. aeruginosa strains that form three clades represented by strain PA14, PA01 and PA7, respectively. Bootstrap values $>75 \%$ are shown.

expression of virulence factors $[15,17,18,30]$, and our own analysis confirmed that an array of genes involved in phosphate acquisition and lipid remodelling in $P$. aeruginosa are indeed highly expressed in sputum samples from the lung microbiome of CF patients (Fig. 2d). Polymyxins represent the drug-of-last resort for effectively treating carbapenem-resistant $P$. aeruginosa infections $[3,39]$.

To test the sensitivity of the mutants in the PlcP-Agt pathway to polymyxin B, we compared WT and mutants using kill curve analyses as the typically used disk diffusion method does not work efficiently for cationic antimicrobials [40]. Indeed, there was a significant decrease in the survival of all three PAO1 glycolipid synthesis mutants $(\Delta p l c P, \Delta a g t 1$ and $\Delta a g t 2)$ compared to the wild type when challenged with polymyxin $\mathrm{B}$, suggesting a protective role of glycolipids in polymyxin B resistance (Fig. 5c). Such a protective role of glycolipids in polymyxin B resistance was not observed for other antibiotics, including ciprofloxacin, gentamicin, ceftazidime and meropenem (data not shown). P. aeruginosa is known to enhance its resistance to polymyxins through decoration of its LPS layer using either 4-amino-4-deoxy-Larabinose (L-Ara4N) by $\operatorname{arnB}$ [5], or the addition of phosphoethanolamine (pEtN) by eptA [6]. It is thought that these changes perturb the electrostatic interaction between the cationic polymyxin B and the normally negatively charged LPS. To investigate whether these mechanisms play a role in the glycolipid-deficient mutants, we conducted a comparative proteomics analysis of the $\Delta p l c P$ mutant and WT under 
(a)

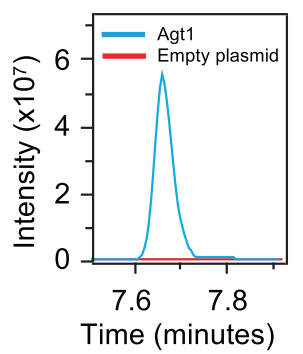

(b)

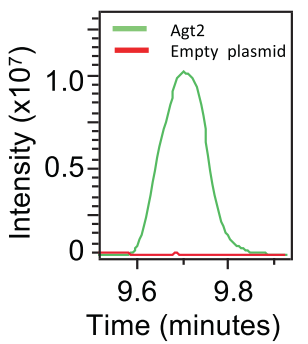

MGDG in recombinant $E$. coli

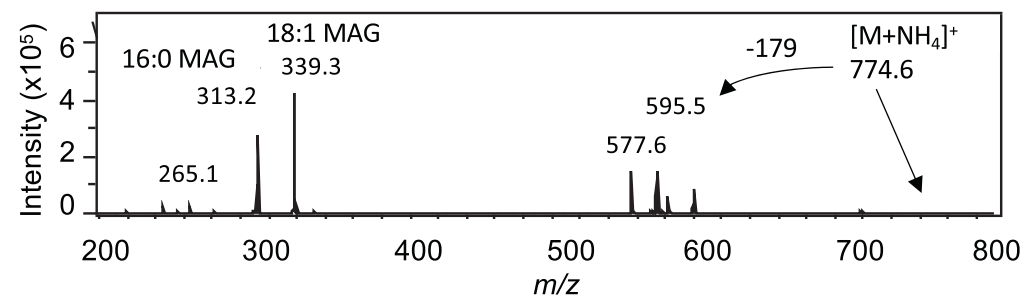

GADG in recombinant $E$. coli

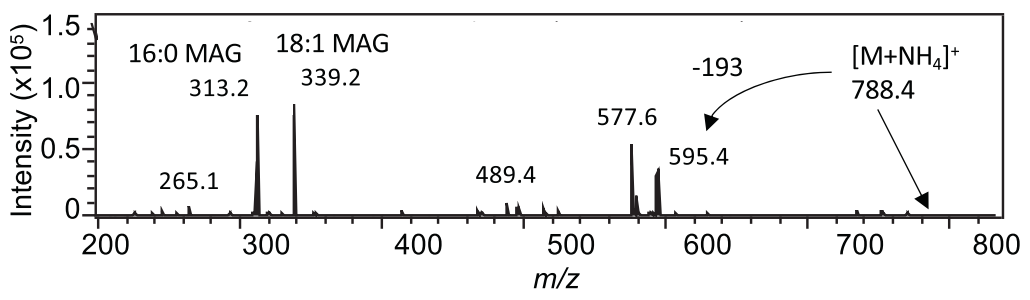

(c)

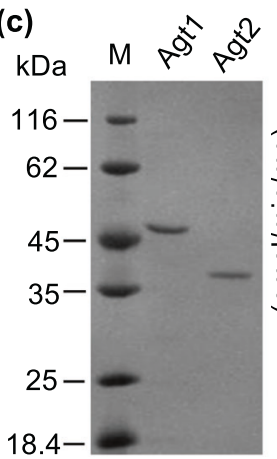

Agt1

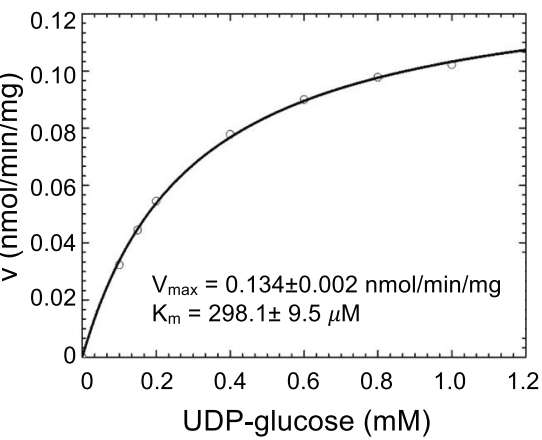

Agt2

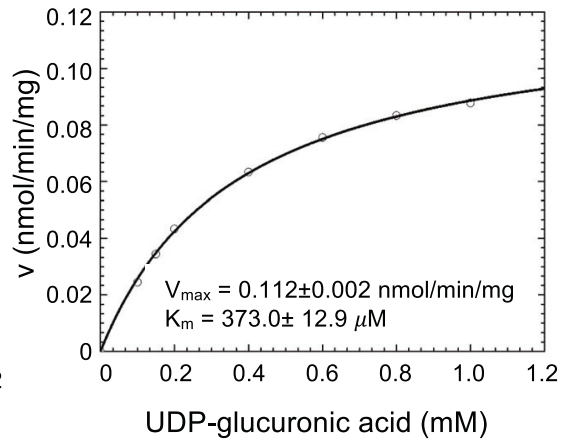

(d)

MGDG 16:0/18:1 M 756.3

GADG 16:0/18:1 M 770.4

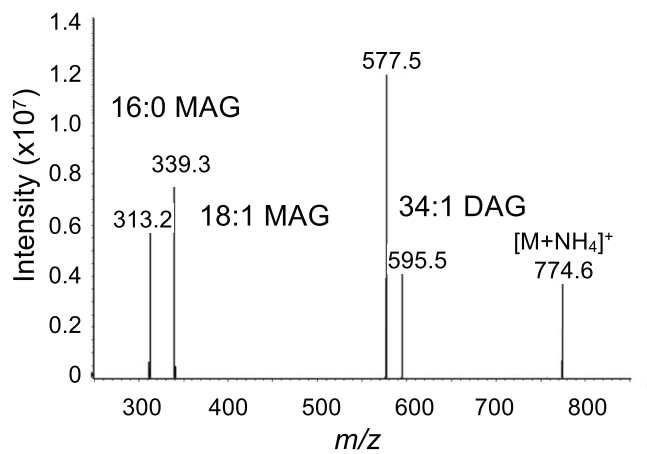

Fig. 4 Characterisation of glycolipid formation from recombinant Agt1 and Agt2. a Extracted ion chromatogram of the MGDG lipid from recombinant $E$. coli expressing Agt1. An empty vector control is also shown (red line). The identity of MGDG is further validated using mass spectrometry fragmentation showing the neutral loss of 179 corresponding to the loss of glucose and the formation of monoacylglycerols (MAG) with C16:0 or C18:1 ( $\mathrm{m} / \mathrm{z}, 313.2,339.3)$. b Extracted ion chromatogram of the GADG lipid from recombinant $E$. coli expressing Agt2. An empty vector control is also shown (red line). The identity of GADG is further validated using mass spectrometry

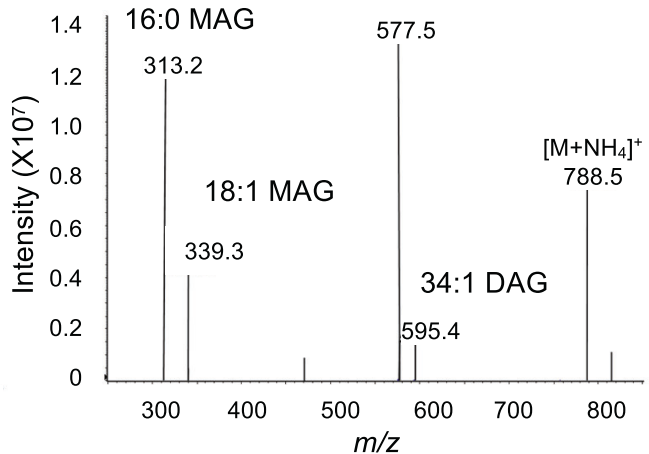

fragmentation showing the neutral loss of 193 corresponding to the loss of glucose and the formation of monoacylglycerols (MAG) with C16:0 or C18:1 ( $\mathrm{m} / \mathrm{z}, 313.2,339.2)$. c Purified Agt1 and Agt2 protein from recombinant $E$. coli (left panel) and Michaelis Menten kinetics of Agt1 towards UDP-glucose (middle panel) and Agt2 towards UDPglucuronic acid (right panel) as substrate, respectively. d Mass spectrometry identification of MGDG and GADG produced from purified Agt1 and Agt2 using DAG and UDP-glucose and UDP-glucuronic acid as the substrate, respectively. 
(a)
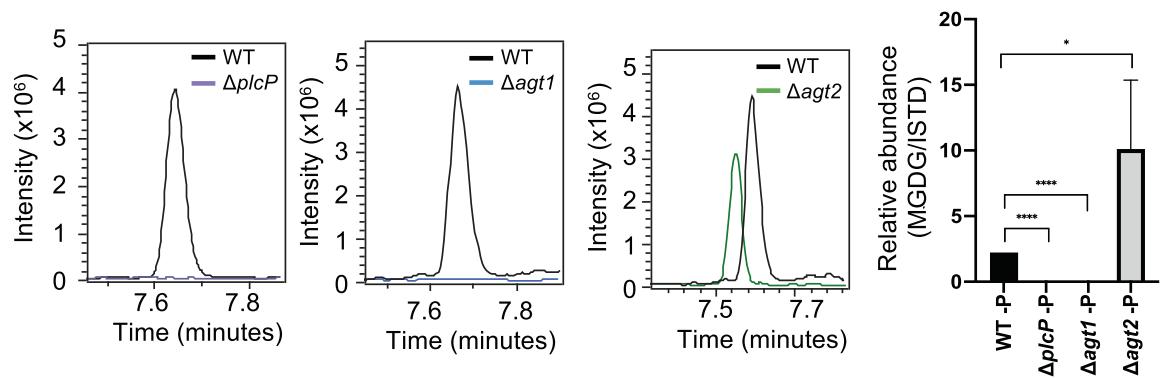

(b)
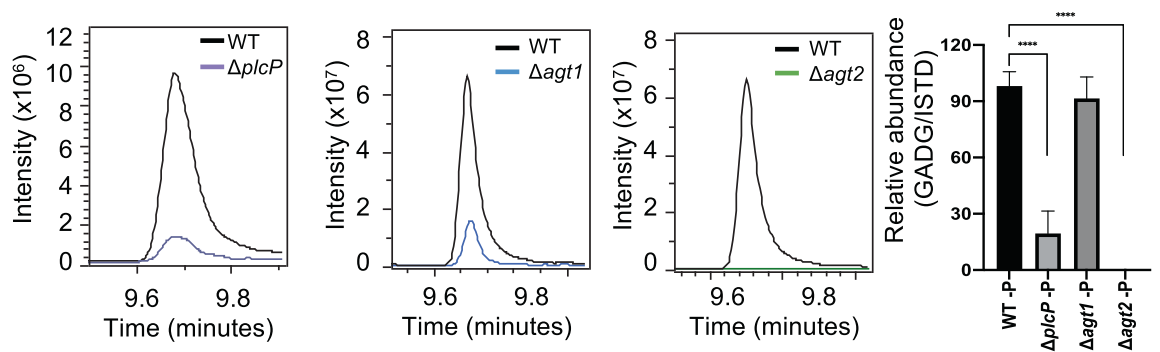

(c)

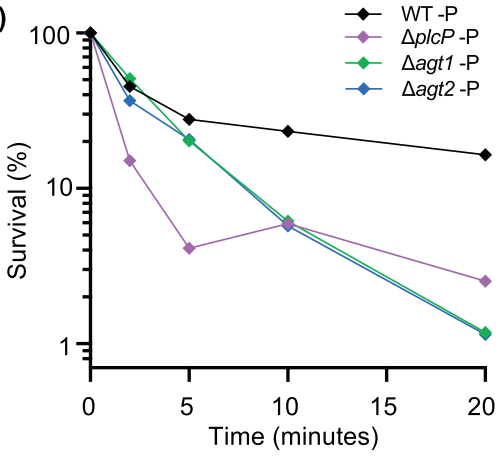

(d)

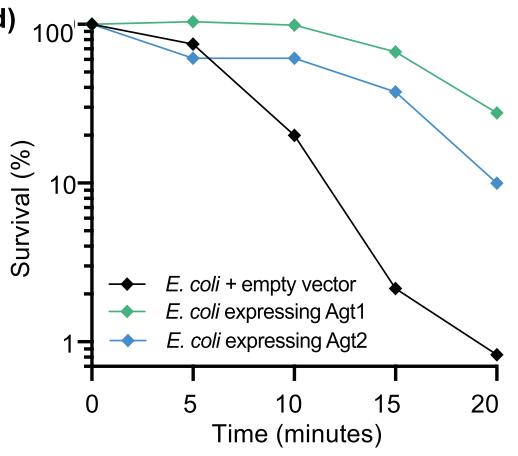

Fig. 5 Glycolipid formation in $P$. aeruginosa and mutants under $\mathbf{P i}$ stress, showing a protective role of glycolipids to polymyxin $B$. Relative abundance of the glycolipid MGDG (a) and GADG (b) in $P$. aeruginosa mutants $p l c P$ (purple trace), agt1 (blue trace) and agt 2 (green, trace) compared to the wild type (WT). Cells were cultivated under low $\mathrm{Pi}$ conditions $(50 \mu \mathrm{M})$ and a representative extracted ion chromatogram of MGDG/GADG is shown between the WT, (black trace) and each mutant. The right most panel shows the abundance of MGDG or GADG calculated relative to an internal lipid standard d17:1/12:0 sphingosylphosphoethanolamine (Sigma-Aldrich) in the wild-type and mutant strains of $P$. aeruginosa. Values are calculated from three biological replicates and the error bars denote standard

Pi-deplete conditions, which revealed only a small number of differentially expressed proteins (Table S3). The majority of these differentially expressed proteins are uncharacterised. However, importantly, LPS modification enzymes previously found to confer antimicrobial peptide resistance, such as ArnB and EptA, were not differentially expressed between the WT and $\Delta p l c P$ mutant. Therefore, our data suggests that it is the glycolipids that are the major contributor to increased polymyxin $\mathrm{B}$ resistance, which constitutes a new biological deviation. MGDG, monoglucosyldiacylglycerol, GADG, glucuronic acid-diacylglycerol. c Survival of glycolipid remodelling mutants $\Delta p l c P$ (purple), $\Delta a g t l$ (green) and $\Delta a g t 2$ (blue) when challenged with $4 \mu \mathrm{g} \mathrm{mL}^{-1}$ polymyxin B compared to WT under Pi stress (black). All experiments were conducted under Pi stress conditions and the results are the average of three biological replicates; error bars denote standard deviation. d Survival of glycolipid producing Escherichia coli when challenged with $20 \mu \mathrm{g} \mathrm{mL} \mathrm{m}^{-1}$ polymyxin B. All experiments were conducted in three replicates and error bars denote standard deviation. Black, E. coli containing the empty vector pET28a; green, E. coli containing plasmid pET28a-Agt1; blue, E. coli containing plasmid pET28a-Agt2.

mechanism for polymyxin resistance. To this end, we tested the resistance to polymyxin B of recombinant $E$. coli strains overexpressing $P$. aeruginosa Agt1 and Agt2, that produce MGDG and GADG, respectively (Fig. 4a, b). Indeed, in this gain-of-function assay, both Agt1 and Agt2-overexpressing E. coli strains had enhanced resistance to polymyxin B compared to the empty vector control (Fig. 5d), supporting the protective role of these glycolipids to antimicrobial peptides. 
To conclude, we present here the discovery of novel glycolipids produced in $P$. aeruginosa during adapation to phosphorus stress. This lipid renovation pathway is strictly conserved in all $P$. aeruginosa isolates to date and highly expressed in the metatranscriptome of $\mathrm{CF}$ patients, suggesting a key role of lipid remodelling in the ecophysiology of this bacterium. Interestingly, lipid remodelling as a response to survive phosphorus stress in turn comes with trade-offs in terms of antibiotic resistance; these glycolipids may protect the bacterium from insult by cationic antimicrobial peptides, highlighting a new resistance mechanism to polymyxin B which has been previously overlooked. It remains to be seen whether the altered susceptibility to polymyxin B is the sole trade-off following lipid remodelling of phospholipids to glycolipids. After all, evolution appears to have selected phospholipids as the dominant lipids in the last universal common ancestor [12].

Acknowledgements This work was funded by an MRC Doctoral Training Partnership studentship in Interdisciplinary Biomedical Research (MR/J003964/1) awarded to RAJ and by a Royal Society International Exchanges 2017 Cost Share (China) award (IECINSFC 170213; grant agreement no. 170213). AM and YC are supported by a European Research Council (ERC) award under the European Union's Horizon 2020 research and innovation programme (grant agreement no. 726116). We also thank the Proteomics Research Technology Platform, University of Warwick, UK for their contribution.

\section{Compliance with ethical standards}

Conflict of interest The authors declare no competing interests.

Publisher's note Springer Nature remains neutral with regard to jurisdictional claims in published maps and institutional affiliations.

Open Access This article is licensed under a Creative Commons Attribution 4.0 International License, which permits use, sharing, adaptation, distribution and reproduction in any medium or format, as long as you give appropriate credit to the original author(s) and the source, provide a link to the Creative Commons license, and indicate if changes were made. The images or other third party material in this article are included in the article's Creative Commons license, unless indicated otherwise in a credit line to the material. If material is not included in the article's Creative Commons license and your intended use is not permitted by statutory regulation or exceeds the permitted use, you will need to obtain permission directly from the copyright holder. To view a copy of this license, visit http://creativecommons. org/licenses/by/4.0/.

\section{References}

1. Murray TS, Egan M, Kazmierczak BI. Pseudomonas aeruginosa chronic colonization in cystic fibrosis patients. Curr Opin Pediatr. 2007;19:83-8.

2. Gaynes R, Edwards JR, System NNIS. Overview of nosocomial infections caused by Gram-negative bacilli. Clin Infect Dis. 2005;41:848-54.

3. Hawkey PM, Livermore DM. Carbapenem antibiotics for serious infections. BMJ. 2012;344:e3236.
4. Landman D, Georgescu C, Martin DA, Quale J. Polymyxins revisited. Clin Microbiol Rev. 2008;21:449-65.

5. Chung ES, Lee JY, Rhee JY, Ko KS. Colistin resistance in Pseudomonas aeruginosa that is not linked to arnB. J Med Microbiol. 2017;66:833-41.

6. Nowicki EM, O'Brien JP, Brodbelt JS, Trent MS. Extracellular zinc induces phosphoethanolamine addition to Pseudomonas aeruginosa lipid A via the ColRS two-component system. Mol Microbiol. 2015;97:166-78.

7. Parsons JB, Rock CO. Bacterial lipids: metabolism and membrane homeostasis. Prog Lipid Res. 2013;52:249-76.

8. Zhang Y-M, Rock CO. Membrane lipid homeostasis in bacteria. Nat Rev Microbiol. 2008;6:222-33.

9. van Meer G, Voelker DR, Feigenson GW. Membrane lipids: where they are and how they behave. Nat Rev Mol Cell Biol. 2008;9:112-24.

10. Harayama T, Riezman H. Understanding the diversity of membrane lipid composition. Nat Rev Mol Cell Biol. 2018;19:281-96.

11. May KL, Silhavy TJ. Making a membrane on the other side of the wall. Biochim et Biophy Acta. 2017;1862:1386-93.

12. Lombard J, López-García P, Moreira D. The early evolution of lipid membranes and the three domains of life. Nat Rev Microbiol. 2012;10:507-15

13. Peretó J, López-García P, Moreira D. Ancestral lipid biosynthesis and early membrane evolution. Trends Biochem Sci. 2004;29:469-77.

14. Sahonero-Canavesi DX, López-Lara IM, Geiger O. Membrane lipid degradation and lipid cycles in microbes. Aerob Utilization Hydrocarb Oils Lipids. 2019. https://doi.org/10.1007/978-3-31950418-6_38.

15. Lamarche MG, Wanner BL, Crépin S, Harel J. The phosphate regulon and bacterial virulence: a regulatory network connecting phosphate homeostasis and pathogenesis. FEMS Microbiol Rev. 2008;32:461-73.

16. Long J, Zaborina O, Holbrook C, Zaborin A, Alverdy J. Depletion of intestinal phosphate after operative injury activates the virulence of $P$. aeruginosa causing lethal gut-derived sepsis. Surgery. 2008;144:189-97.

17. Francis VI, Stevenson EC, Porter SL. Two-component systems required for virulence in Pseudomonas aeruginosa. FEMS Microbiol. Lett. 2017;364. https://doi.org/10.1093/femsle/fnx104.

18. Bains M, Fernández L, Hancock REW. Phosphate starvation promotes swarming motility and cytotoxicity of Pseudomonas aeruginosa. Appl Environ Microbiol. 2012;78:6762-8.

19. Son MS, Matthews WJ, Kang Y, Nguyen DT, Hoang TT. In vivo evidence of Pseudomonas aeruginosa nutrient acquisition and pathogenesis in the lungs of cystic fibrosis patients. Infect Immun. 2007;75:5313-24.

20. Lidbury IDEA, Murphy ARJ, Scanlan DJ, Bending GD, Jones AME, Moore JD, et al. Comparative genomic, proteomic and exoproteomic analyses of three Pseudomonas strains reveals novel insights into the phosphorus scavenging capabilities of soil bacteria. Environ Microbiol. 2016;18:3535-49.

21. Sebastián M, Smith AF, González JM, Fredricks HF, Van Mooy B, Koblížek M, et al. Lipid remodelling is a widespread strategy in marine heterotrophic bacteria upon phosphorus deficiency. ISME J. 2016;10:968-78.

22. Smith AF, Rihtman B, Stirrup R, Silvano E, Mausz MA, Scanlan $\mathrm{DJ}$, et al. Elucidation of glutamine lipid biosynthesis in marine bacteria reveals its importance under phosphorus deplete growth in Rhodobacteraceae. ISME J. 2019;13:39-49.

23. Diercks H, Semeniuk A, Gisch N, Moll H, Duda KA, Hölzl G. Accumulation of novel glycolipids and ornithine lipids in Mesorhizobium loti under phosphate deprivation. J Bacteriol. 2015;197:497-509.

24. Shropshire H, Jones RA, Aguilo-Ferretjans MM, Scanlan DJ, Chen Y. Proteomics insights into the Burkholderia cenocepacia 
phosphorus stress response. Environ Microbiol. 2021. https://doi. org/10.1111/1462-2920.15451.

25. Bailey TL, Elkan C. Fitting a mixture model by expectation maximization to discover motifs in biopolymers. Proceedings of the Second International Conference on Intelligent Systems for Molecular Biology. Menlo Park, Californias: AAAI Press; 1994. p. 28-36.

26. Cobián Güemes AG, Lim YW, Quinn RA, Conrad DJ, Benler S, Maughan $\mathrm{H}$, et al. Cystic fibrosis rapid response: translating multi-omics data into clinically relevant information. mBio. 2019;16:e00431-19.

27. Cornforth DM, Dees JL, Ibberson CB, Huse HK, Mathiesen IH, Kirketerp-Moller K, et al. Pseudomonas aeruginosa trascriptome during human infection. Proc Nat Acad Sci USA. 2018;115: E5125-34.

28. Jones HJ, Krober E, Stephenson J, Mausz MA, Jameson E, Millard A, et al. A new family of uncultivated bacteria involved in methanogenesis from the ubiquitous osmolyte glycine betaine in coastal saltmarsh sediments. Microbiome. 2019;7:120.

29. Kim SK, Park SJ, Li XH, Choi YS, Im DS, Lee JH. Bacterial ornithine lipid, a surrogate membrane lipid under phosphate-limiting conditions, plays important roles in bacterial persistence and interaction with host. Environ Microbiol. 2018;20:3992-4008.

30. Lewenza S, Falsafi R, Bains M, Rohs P, Stupak J, Sprott GD, et al. The olsA gene mediates the synthesis of an ornithine lipid in Pseudomonas aeruginosa during growth under phosphate-limiting conditions, but is not involved in antimicrobial peptide susceptibility. FEMS Microbiol Lett. 2011;320:95-102.

31. Wilton M, Halverson TW, Charron-Mazenod L, Parkins MD, Lewnza S. Secreted phosphatase and deoxyribonuclease are required by Pseudomonas aeruginosa to defend against neutrophil extracellular traps. Infect Immun. 2018;86:e00403-18.

32. Wei T, Quareshy M, Zhang YZ, Scanlan DJ, Chen Y. Manganese is essential for PlcP metallophosphoesterase activity involved in lipid remodeling in abundant marine heterotrophic bacteria. Appl Environ Microbiol 2018;84:e01109-18.

33. Zavaleta-Pastor, M, Sohlenkamp, C, Gao, J-L, Guan, Z, Zaheer, R, Finan, TM, et al. Sinorhizobium meliloti phospholipase C required for lipid remodeling during phosphorus limitation. Proc Natl Acad Sci USA. 2010;107:302-7.

34. Freschi L, Jeukens J, Kukavica-Ibrulj I, Boyle B, Dupont MJ, Laroche J, et al. Clinical utilization of genomics data produced by the international Pseudomonas aeruginosa consortium. Front Microbiol. 2015;29:1036.

35. Ozer EA, Nnah E, Didelot X, Whitaker RJ, Hauser AR. The population structure of Pseudomonas aeruginosa is characterized by genetic isolation of exoU+ and exoS + lineages. Genome Biol Evol. 2019;11:1780-96.

36. Chugani SA, Greenberg EP. The influence of human respiratory epithelia on Pseudomonas aeruginosa gene expression. Microb Pathog. 2007;42:29-35.

37. Frisk A, Schurr JR, Wang G, Bertucci DC, Marrero L, Hwang SH, et al. Transcriptome analysis of Pseudomonas aeruginosa after interaction with human airway epithelial cells. Infect Immun. 2004;72:5433-8.

38. Filloux A, Bally M, Soscia C, Murgier M, Lazdunski A. Phosphate regulation in Pseudomonas aeruginosa: cloning of the alkaline phosphatase gene and identification of $p h o B$ and $p h o R$ like gnes. Mol Gen Genet. 1988;212:510-3.

39. Poirel L, Jayol A, Nordmann P. Polymyxins: antibacterial activity, susceptibility testing, and resistance mechanisms encoded by plasmids or chromosomes. Clin Microbiol Rev. 2017;30:557-96.

40. Ezadi F, Ardebili A, Mirnead R. Antimicrobial susceptibility testing for polymyxins: challenges, issues, and recommendations. J Clin Microbiol. 2019;57:e01390-18.

41. Semeniuk A, Sohlenkamp C, Duda K, Hölzl G. A bifunctional glycosyltransferase from Agrobacterium tumefaciens synthesizes monoglucosyl and glucuronosyl diacylglycerol under phosphate deprivation. J Biol Chem. 2014;289:10104-14.

42. Monds RD, Newell PD, Schwartzman JA, O'Toole GA. Conservation of the Pho regulon in Pseudomonas fluorescens Pf0-1. Appl Environ Microbiol. 2006;72:1910-24.

43. Minh BQ, Schmidt HA, Chernomor O, Schrempf D, Woodhams MD, von Haeseler A, et al. IQ-TREE 2: New models and efficient methods for phylogenetic inference in the genomic era. Mol Biol Evol. 2020;37:1530-4. 